\title{
Outsourced hearing in spiders by using their webs as auditory sensors
}

\section{Abstract}

Hearing is a fundamental sense of many animals, including all mammals, birds, some reptiles, amphibians, fish and arthropods ${ }^{1,2}$. The auditory organs of these animals are extremely diverse in anatomy after hundreds of millions of years of evolution ${ }^{3-5}$, yet all are made up of cellular tissue and are embodied meaning that its functional anatomy is constrained by developmental morphogenesis. Here we show hearing in the orb-weaving spider, Larinioides sclopetarius is not constrained by embodiment but is extended through outsourcing hearing to its proteinaceous, selfmanufactured orb-web, and hence under behavioral control, not developmental biology. We find the wispy, wheel-shaped orb-web acts as a hyperacute acoustic array to capture the sound-induced air particle movements that approach the maximum physical efficiency, better than the acoustic responsivity of all previously known ears ${ }^{6,7}$. By manipulating the web threads with its eight vibration-sensitive $\operatorname{legs}^{8-10}$, the spider remotely detects and localizes the source of an incoming airborne acoustic wave emitted by approaching prey or predators. By outsourcing its acoustic sensors to its web, the spider is released from embodied morphogenetic constraints and permits the araneid spider to increase its sound-sensitive surface area enormously, up to 10,000 times greater than the spider itself ${ }^{11}$. The use of the web also enables a spider the flexibility to functionally adjust and regularly regenerate its "external ear" according to its needs. This finding opens a new perspective on animal hearing - the "outsourcing" and "supersizing" of auditory function in a spider, one of the earliest animals to live on land ${ }^{12}$. The novel hearing mechanism provides unique features for studying extended and regenerative sensing ${ }^{13-15}$, and designing novel acoustic flow detectors for precise fluid dynamic measurement and manipulation ${ }^{16-18}$.

During the water-to-land transition, animals have gone through dramatic challenges in aerial hearing ${ }^{3,4}$. To effectively detect weak, distant airborne sound, terrestrial vertebrates and some invertebrates have evolved the tympanic eardrums which are very sensitive to the pressure component of sound ${ }^{3,19}$. 
Alternatively, some arthropods, especially those of miniscule size, have evolved pendulum-like, long wispy filaments to detect the velocity component of sound ${ }^{20-22}$. While the auditory organs of different animals are extremely diverse in anatomy after hundreds of millions of years of evolution, they are all organs of cellular origin and hence embodied. Hence, they are necessarily constrained by morphogenesis.

Spiders are among the oldest land animals, with a fossil record dating back to the Devonian Period (around 380-million years ago) ${ }^{12}$. All spiders produce silk, a biomaterial that can be stronger than steel in strength-to-weight ratio yet extremely flexible ${ }^{23}$, owing to its exceptional material properties. When woven into a broad latticework, a web can serve as a net for capturing prey that fly or walk into it ${ }^{24-26}$. We previously showed that a single strand of nano-dimensional spider silk can move with a velocity very close to that of the surrounding air particle movements, with a flat frequency response from infrasound to ultrasound, despite the low viscosity and low density of air ${ }^{7}$. Here we show that the highly responsive aerodynamic property of silk fibers are woven and stretched into diaphanous orb-web can function as a huge acoustic array "antenna," which allows the spider to efficiently detect faint airborne sound from a distant source. This outsourced web-array "ear" operates on a very different principle from the much smaller embodied auditory organs of all other animals.

We have found that the orb-weaving spiders detect and localize distant airborne sound (Fig. 1, Supplementary Video 1, Supplementary Video 2). Spiders used in this study are Larinioides sclopetarius, a familiar araneid related to the species celebrated by E.B. White, in "Charlotte's Web". All spiders were field-collected in Vestal, N.Y. and kept in laboratory conditions, where they spontaneously spin orb-webs within wooden frames $(30 \mathrm{~cm} \times 30 \mathrm{~cm} \times 1 \mathrm{~cm})$. We videotaped spider behavioral reactions $(\mathrm{N}=60$ spiders $)$ to airborne acoustic tones within a spacious closed anechoic room with a $60 \mathrm{fps}$ video camera, in 2 different auditory configurations (see Extended Data Fig. 1, and Supplementary Table 1): 1) normalincident sound waves to the orb-web plane, emitted from a frontally-positioned loudspeaker, $3.0 \mathrm{~m}$ away to the spider; and 2) oblique-incident directional sound waves, emitted from loudspeakers placed at 45 degrees to the left and right of the spider in azimuth, $0.5 \mathrm{~m}$ away. Before making any measurements, we ensured that the spiders were undisturbed and resting naturally within the hub region. Each spider was acoustically stimulated only once per trial. The behavior of each spider was video-monitored for 5 seconds after initiating the stimulus tone. Its behavior in the 5 seconds preceding acoustic stimulation served as the silent control period $(0 \mathrm{~dB})$. Within our sealed chamber there were no uncontrolled airflows or substrate vibrations (Extended Data Fig. 2) to disturb the spider; the airborne stimuli from the speakers were the only source of web vibrations.

The responsiveness and sensitivity of spiders to relatively distant $(3.0 \mathrm{~m})$ normal-incident acoustic waves are shown in Fig. 1a-d. Four distinct behavioral responses, in the form of rapid adaptations in body posture, were provoked by acoustic stimulation under 4 combinations of tone frequency ( $200 \mathrm{~Hz}$ and 1000 $\mathrm{Hz}$ ) and sound pressure level (SPL, a "soft" $68 \mathrm{~dB}$ and an "intense" $88 \mathrm{~dB}$ ). We assigned 48 spiders to 4 groups ( $\mathrm{N}=12$ spiders in each group). The duration of each tone stimulus, at a given SPL and frequency, was 3 seconds. Of 12 individuals tested in each group, 6/12 and 11/12 responded to the $200 \mathrm{~Hz}$ tones at $68 \mathrm{~dB}$ and $88 \mathrm{~dB}$ respectively, $3 / 12$ and 9/12 responded to the $1000 \mathrm{~Hz}$ tones to $68 \mathrm{~dB}$ and $88 \mathrm{~dB}$ sound levels, respectively, but none responded during silent controls. We identified several categories of intensity-dependent behaviors to $200 \mathrm{~Hz}$ tones. At high $88 \mathrm{~dB}$ levels (Fig. 1b, Supplementary Video 1), bodily responses consisted of 1) crouching (pulling the web strands slightly with legs), 2) stretching-out or flattening of the body (rapidly extending all 8 legs outward from the cephalothorax), 3) foreleg displaying (lifting the forelegs into the air), and 4) turning (abruptly changing the direction of the body). The response to the $88 \mathrm{~dB}$ stimuli was complex and variable-some spiders responded sequentially with 
several behaviors, so we only recorded the spider's initial response for labeling the behavior. Importantly, the only behavioral response to the low intensity stimulation, at $68 \mathrm{~dB}$ was crouching (Fig. 1c).

We observed that spiders can localize the direction of airborne sound accurately. Active and rapid turning movements toward the source speaker were observed when the speaker location was shifted from normal to oblique incident $\left(45^{\circ}, \mathrm{L} / \mathrm{R}\right)$ in azimuth (Fig. 1e, f, Supplementary Video 2). Unlike normalincident soundwaves that arrive at the orb-web plane simultaneously, oblique-incident soundwaves arrive with brief delays, creating directional acoustic cues such as time, amplitude and phase differences at different locations of the orb-web ${ }^{28}$. Of 12 individuals tested for directional hearing, all (12/12, Fig. 1e) responded to oblique-incident $200 \mathrm{~Hz}$ tones at $88 \mathrm{~dB}$ (Extended Data Fig. 1d), similar to the high responsivity (11/12, Fig. 1b) to the normal-incident sound at $88 \mathrm{~dB}$ from the front. However, more spiders (5/12, Fig. 1e, f) responded to the directional oblique-incident sound by turning towards the stimulating speaker, compared to only 1 of 12 spiders (Fig. 1b) that turned in response to normal-incident sound.

Having found that L. sclopetarius spiders exhibit behavioral responses by detecting and localizing airborne sound, we then investigated the physical properties of the web as an acoustic array by measuring the mechanical response of the web to direct acoustic stimulation using sounds that are salient to spider hearing (Fig. 2, Supplementary Video 3, Supplementary Text). Using Doppler vibrometry, we measured sound-induced out-of-plane web motion for the web alone and with live spiders resting in the web's hub. The web was separated from a loudspeaker $3.0 \mathrm{~m}$ away and aligned so that the direction of sound propagation was perpendicular to the plane of the orb-web, creating a normal-incident planar sound wave (Extended Data Fig. 1e, f). We broadcast sinusoidal test tones ranging from $100 \mathrm{~Hz}$ to $10,000 \mathrm{~Hz}$. The air particle velocity component, $\mathrm{u}(\mathrm{t})$, of a sound wave was computed from the measurement of the pressure $p(t)$ using a calibrated pressure-sensitive microphone near, but not touching the web, where $u(t)=p(t) / \rho_{0} c$, $\rho_{0}$ is the density of air, $\mathrm{c}$ is the speed of sound in air ${ }^{29}$. Fig. 2a shows an example of the measured orb-web response to $200 \mathrm{~Hz}$ acoustic signals, in which the orb-web follows the air particle motion with almost full fidelity and maximum physical efficiency (i.e. $\mathrm{V}_{\text {web }} / \mathrm{V}_{\text {air }} \sim 1$ ), better than the acoustic responsivity of all known ears ${ }^{6,7}$. Similarly, at other measurement frequencies, orb-webs responded effectively to airborne acoustic signals, a bandwidth encompassing the sounds produced by potential prey and predators, such as insects and birds (Fig 2c, e, Supplementary Audio 1, Extended Data Fig. 3, 4). The differences in velocity between the spider body and the web (Fig. 2d, e) suggest that mechanical strain is actively induced on the spider's legs when stimulated by airborne sound. In spiders, vibrational signals such as faint acoustic stimuli are presumably detected by the strain-sensitive lyriform organs located in spider legs ${ }^{8-10}$.

It is important to note that some spider species and insects can detect air particle velocity with pendulum-like, long, wispy cuticular hair receptors ${ }^{20-22}$. It is also widely known that spiders can sense movements of the web when a vibrating stimulus is applied directly (touching) to the web silk $\mathrm{k}^{25-27}$. To determine whether the spider is detecting highly circumscribed sound-induced web movements or is directly detecting some fluctuating acoustic quantity of the air (such as pressure or velocity) through some unidentified mechanosensor, we stimulated only small, focal regions of the web while ensuring that any airborne acoustic signal would have such a low amplitude that it would not be detectable by the spider, perched in the center of the web, distant from such a focal acoustic stimulus.

To accomplish this, we used a miniature speaker (dimensions $15 \mathrm{~mm} \times 11 \mathrm{~mm} \times 3 \mathrm{~mm}$ ) as a focal sound source (Fig. 3a). The speaker was positioned $50 \mathrm{~mm}$ in radial distance away from the spider, that was resting in the web's hub. By aligning the small speaker as near as possible to the web without actually touching it, we created a localized "near-field" sound causing oscillations in air particle velocity, from the mini-speaker, but which rapidly decayed with distance as it propagates through the air (Fig. 3b, c). We showed that the near-field airborne stimulation generated by the mini-speaker attenuated quickly with 
distance, and fell well below the spider's detection threshold after it spread to distantly perched spider $(<50 \mathrm{~dB}$, see Fig. 3b, c and Extended Data Fig. 5). However, the out-of-plane web movements induced by the airborne sound created by the mini-speaker attenuated less, so that vibrational signals were transmitted to the spider. Results show that the spiders perceived minute localized web vibrations at extremely low intensity levels (Fig. 3d, e). Of 12 individuals, 4 responded to $200 \mathrm{~Hz}$ web vibration tones of $3 \mathrm{~s}$ duration with equivalent $\mathrm{SPL} \leq 68 \mathrm{~dB}\left(\mathrm{~V}_{\mathrm{rms}} \leq 0.12 \mathrm{~mm} / \mathrm{s}\right)$. Spiders responded to these minute web vibrations by crouching, just as they respond to airborne stimuli at $68 \mathrm{~dB}$ from a loudspeaker $3 \mathrm{~m}$ away (Fig. 1c, d). The behavioral response of spiders to minute web vibrations induced by the focal airborne sound confirms their abilities to perceive airborne acoustic signals solely by detecting web movements. Given a webenabled hearing threshold lower than $68 \mathrm{~dB}$ (Fig. 3d), orb-weaving spiders should be able to detect and localize predators and prey such as birds and crickets at a distance more than $10 \mathrm{~m}$ away, considering the sound loudness produced by distant animals (Supplementary Table 2).

Outsourcing the acoustic sensors to its web provides the spider with the flexibility to adjust its hearing adaptively according to its needs. When an orb-web is torn or badly damaged that disrupts its radial symmetry, an orb-weaver can recover its hearing through the acoustic array by weaving a new one within an hour. By adjusting web geometries and pre-tensions during the web weaving, the spider is able to adjust both the level and tuning of the mechanical input from the web threads to the metatarsal organ (See Supplementary Text and Extended Data Fig. 6). For example, a variably-tensioned orb-web could efficiently filter out the bio-irrelevant low frequency noises which are unavoidable in the natural environment, such as the wind perturbation which has tremendous velocity and pressure amplitude than that of biorelevant acoustic signals. In principle, the multipodal spider can adaptively tune its hearing in real-time by behaviorally manipulating its extended "virtual eardrum". Each of its 8 legs is endowed with sensitive metatarsal organs that can be extended in all directions from the center of the web, representing "well-connected" nodes in the wheel-shaped network, consisting of smaller local nodes to interface with the web dynamics. On the one hand, the 8 legs are points of sampling and amplification for sensing, and on the other hand they could serve as feedforward controllers by adjusting postures and positions that amplifies directionality and sensitivity actively ${ }^{30,31}$. The size and shape of an orb web can be varied to meet the needs of a spider's sensory and feeding ecology and demonstrates a remarkable level of flexibility in this surprising bioacoustic control system.

Biologists and material scientists are still discovering new properties of spider silk that can be repurposed as a biomaterial and deployed for practical human applications. Here, we demonstrate how a spider web made of nanoscale protein fibers serves as a megascale acoustic airflow sensor, contrasted sharply with all auditory organs made up cellular tissue, and necessarily subjected to morphogenetic constraints. By getting rid of the morphogenetic constraint, the sensory surface area is up-scaled extensively, much as a radio-telescope senses electromagnetic signals from cosmic sources. The web is analogous to an eardrum in other animals, but it senses the velocity of air particles, not its collective pressure. Spider webs are marvels of bio-architecture that greatly extend the spider's capacity to sense and capture prey much larger than the spider itself ${ }^{14,15,32}$. The spiders also have the flexibility to tune and regenerate their hearing by manipulating the orb-webs. The novel hearing mechanism could provide unique features for studying extended and regenerative sensing ${ }^{13-15}$, and presage a new generation of acoustic fluid-flow detectors in the domain of nanoscale biosensors for applications requiring precise fluid dynamic measurement and manipulation ${ }^{16-18}$.

170 1. Fay, R. R. Hearing in vertebrates: A psychophysics databook. (Hill-Fay Associates, 1988). 
2. Albert, J. T. \& Kozlov, A. S. Comparative Aspects of Hearing in Vertebrates and Insects with Antennal Ears. Current Biology 26, R1050-R1061 (2016).

3. Bergeijk, W. A. V. Evolution of the Sense of Hearing in Vertebrates. Am. Zool. 6, 371-377 (1966).

4. Christensen, C. B., Christensen-Dalsgaard, J. \& Madsen, P. T. Hearing of the African lungfish (Protopterus annectens) suggests underwater pressure detection and rudimentary aerial hearing in early tetrapods. J. Exp. Biol. 218, 381-387 (2015).

5. Goepfert, M.C. and Hennig, R.M. Hearing in insects. Annu. Rev. Entomol. 61, 257-276 (2016).

6. Fratzl, P. \& Barth, F. G. Biomaterial systems for mechanosensing and actuation. Nature 462, 442-448 (2009).

7. Zhou, J. \& Miles, R. N. Sensing fluctuating airflow with spider silk. Proc. Natl. Acad. Sci. 114, 1212012125 (2017).

8. Stafstrom, J. A., Menda, G., Nitzany, E. I., Hebets, E. A. \& Hoy, R. R. Ogre-Faced, Net-Casting Spiders Use Auditory Cues to Detect Airborne Prey. Curr. Biol. 30, 5033-5039.e3 (2020).

9. Walcott, C. \& Kloot, W. G. van der. The physiology of the spider vibration receptor. J. Exp. Zool. 141, 191-244 (1959).

10. Barth, F. G. \& Geethabali. Spider vibration receptors: Threshold curves of individual slits in the metatarsal lyriform organ. J. Comp. Physiol. 148, 175-185 (1982).

11. Gregorič, M., Agnarsson, I., Blackledge, T. A. \& Kuntner, M. Darwin's bark spider: giant prey in giant orb webs (Caerostris darwini, Araneae: Araneidae)? J. Arachnol. 39, 287-295 (2011).

12. Shear, W.A., Palmer, J.M., Coddington, J.A. and Bonamo, P.M. A Devonian spinneret: early evidence of spiders and silk use. Science 246: 479-481 (1989).

13. Japyassú, H. F. \& Laland, K. N. Extended spider cognition. Anim. Cogn. 20, 375-395 (2017).

14. Miller, L. E. et al. Sensing with tools extends somatosensory processing beyond the body. Nature 561, 239-242 (2018).

15. Hochberg, L.R., Serruya, M.D., Friehs, G.M., Mukand, J.A., Saleh, M., Caplan, A.H., Branner, A., Chen, D., Penn, R.D. and Donoghue, J.P. Neuronal ensemble control of prosthetic devices by a human with tetraplegia. Nature 442, 164-171 (2006).

16. Nakata, T. et al. Aerodynamic imaging by mosquitoes inspires a surface detector for autonomous flying vehicles. Science 368, 634-637 (2020).

17. Sackmann, E. K., Fulton, A. L. \& Beebe, D. J. The present and future role of microfluidics in biomedical research. Nature 507, 181-189 (2014).

18. Raissi, M., Yazdani, A. \& Karniadakis, G. E. Hidden fluid mechanics: Learning velocity and pressure fields from flow visualizations. Science 367, 1026-1030 (2020).

19. Robert, D., Amoroso, J. and Hoy, R.R. The evolutionary convergence of hearing in a parasitoid fly and its cricket host. Science 258, 1135-1137 (1992).

20. Shamble, P. S. et al. Airborne Acoustic Perception by a Jumping Spider. Curr. Biol. 26, 2913-2920 (2016).

21. Menda, G. et al. The Long and Short of Hearing in the Mosquito Aedes aegypti. Curr. Biol. 29, 709714.e4 (2019).

22. Taylor, C. J. \& Yack, J. E. Hearing in caterpillars of the monarch butterfly (Danaus plexippus). J. Exp. Biol. 222, jeb211862 (2019).

23. Omenetto, F. G. \& Kaplan, D. L. New Opportunities for an Ancient Material. Science 329, 528-531 (2010).

24. Lin, L. H., Edmonds, D. T. \& Vollrath, F. Structural engineering of an orb-spider's web. Nature 373, 146-148 (1995). 
25. Masters, W. M. \& Markl, H. Vibration Signal Transmission in Spider Orb Webs. Science 213, 363365 (1981).

26. Boys, C. V. The Influence of a Tuning-Fork on the Garden Spider. Nature 23, 149-150 (1880).

27. Mortimer, B., Soler, A., Siviour, C. R., Zaera, R. \& Vollrath, F. Tuning the instrument: sonic properties in the spider's web. J. R. Soc. Interface 13, 20160341 (2016).

28. Landolfa, M. A. \& Barth, F. G. Vibrations in the orb web of the spider Nephila clavipes: cues for discrimination and orientation. J. Comp. Physiol. A 179, 493-508 (1996).

29. Miles, R. N. One Dimensional Sound Fields. in Physical Approach to Engineering Acoustics. 35-52 (Springer International Publishing, 2020).

30. Watanabe, T. Web tuning of an orb-web spider, Octonoba sybotides, regulates prey-catching behaviour. Proc. R. Soc. Lond. B Biol. Sci. 267, 565-569 (2000).

31. Nakata, K. Attention focusing in a sit-and-wait forager: a spider controls its prey-detection ability in different web sectors by adjusting thread tension. Proc. R. Soc. B Biol. Sci. 277, 29-33 (2010).

32. Nyffeler, M. \& Altig, R. Spiders as frog-eaters: a global perspective. J. Arachnol. 48, 26-42 (2020).

33. Sound clips: Catalog no. ML 125254, ML 208175 and ML 516988, Macaulay Library at the Cornell Laboratory of Ornithology, Ithaca, NY. 
a
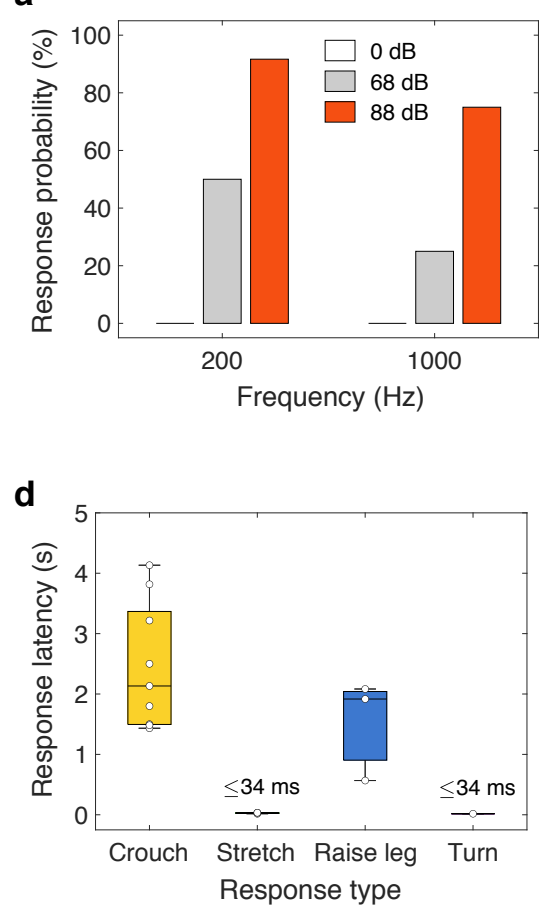

b

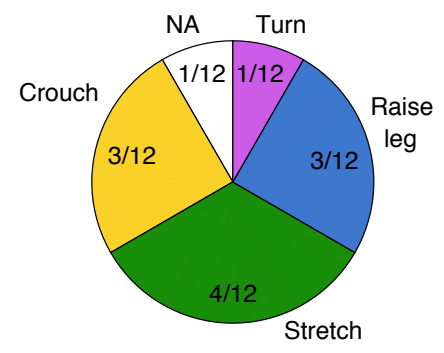

Response type at $88 \mathrm{~dB}$

e

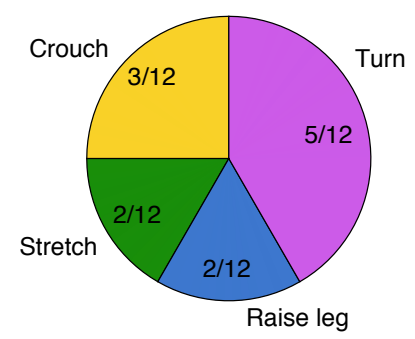

Response type at $88 \mathrm{~dB}$
C

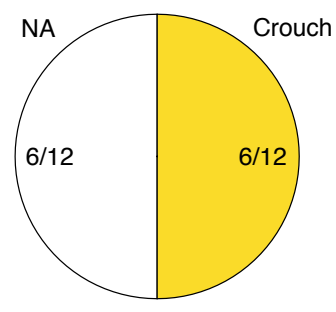

Response type at $68 \mathrm{~dB}$

Fig. 1 | Adaptive behavioral responses made by orb-weaving spiders stimulated by remote airborne sound. Two acoustic hearing conditions of airborne tone stimulation were investigated: 1) normal-incident sound (a-d), generated by a loudspeaker, $3.0 \mathrm{~m}$ distance to the spider, with a propagated direction perpendicular to the plane of the orb-web; 2) oblique-incident sound (e-f), from one of two identical loudspeakers, $0.50 \mathrm{~m}$ distance, randomly placed to the left and right side of the spider, $45^{\circ}$ in azimuth. a, Percentage of spider response ( 4 groups, $\mathrm{N}=12$ spiders in each group) to normal-incident acoustic tones of $3 \mathrm{~s}$ duration. b-d, Behavioral response categories and response latencies (each value, median, interquartile, and range) to $200 \mathrm{~Hz}$ tones. NA represents no behavioral response in the pie charts. e, Response ( $\mathrm{N}=12$ spiders) to oblique-incident acoustic tones $(200 \mathrm{~Hz}, 88 \mathrm{~dB})$. f, Percentage of spiders correctly turning towards the randomly assigned direction of a sound source. 

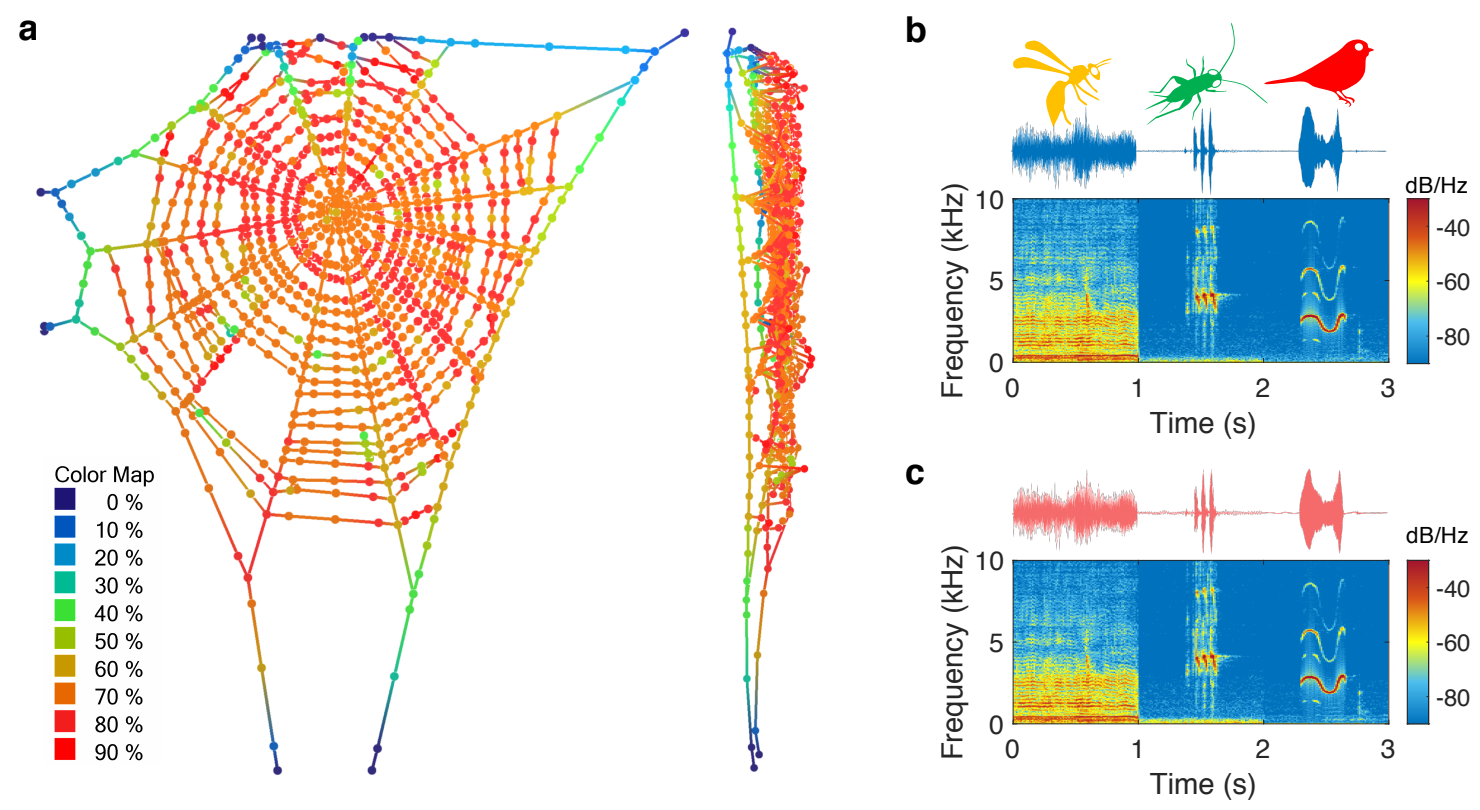

d
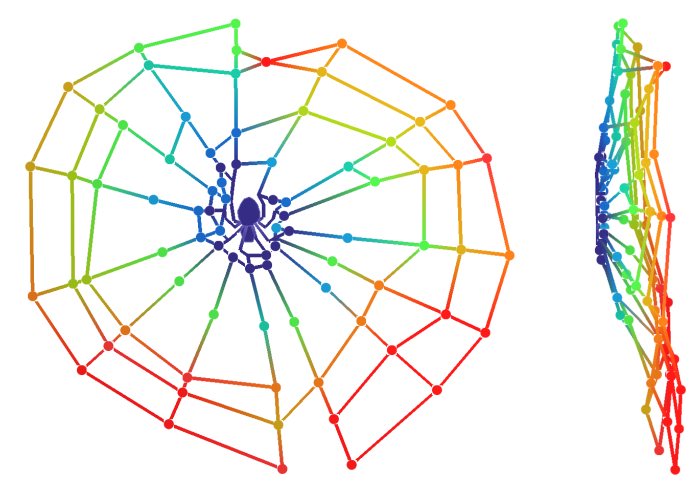

e

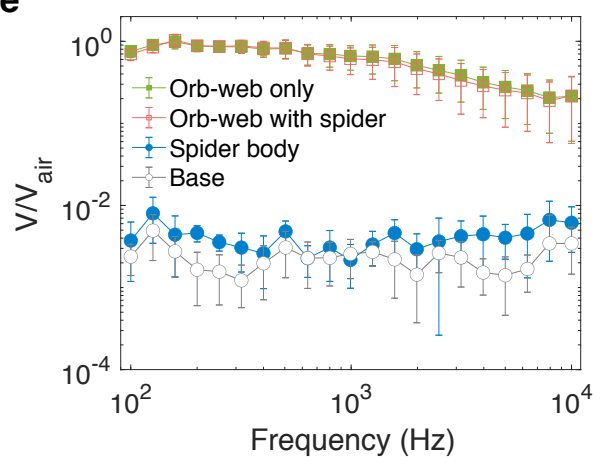

Fig. 2 | Spider orb-web is an enormous, adjustable, regenerative, and highly sensitivity acoustic array without morphogenetic constraints. a-e, Orb-web responses to remote normal-incident sound, generated by a loudspeaker, $3.0 \mathrm{~m}$ distance to the spider. a, Out-of-plane motion of a complete orb-web induced by a $200 \mathrm{~Hz}$ steady-state sound field (see Supplementary Video 1). The colored heat map represents the amplitude ratio of the web thread velocity $\mathrm{V}$ to the air particle velocity $\mathrm{V}_{\text {air, }}$ e.g. $90 \%$ represents $\mathrm{V} / \mathrm{V}_{\text {air }}=0.9 \sim 1.0$. b, c, Time-domain traces and spectrograms of the airborne acoustic signal ${ }^{33}$ measured by a pressure microphone (b), and the signal-induced web motion measured by a laser vibrometer (c) at the hub web position as shown in a. The audio clip of the measured web motion is provided as Supplementary Audio 1. The acoustic signal spans a wide range of frequencies $(100 \mathrm{~Hz}-10000$ $\mathrm{Hz}$ ) including hymenopteran wing-beats, cricket chirpings, and bird songs. d, Heat map depiction of outof-plane motion of web containing a live spider, induced by a $200 \mathrm{~Hz}$ sound field (Supplementary Video 1). Color coding of $\mathbf{d}$ same as a. e, Statistic frequency responses of the spider orb-webs $(\mathrm{N}=12)$ to airborne sound. Individual measurement (1 of 12) contains 1 location on a spider body, 1 location on an orb-web frame base, and 4 locations (up, down, left, and right) on radial threads of an orb-web in radial distance of $5 \mathrm{~cm}$ away from its hub, without and with the spiders resting in the hub webs. Error bars show one standard deviation (SD). 


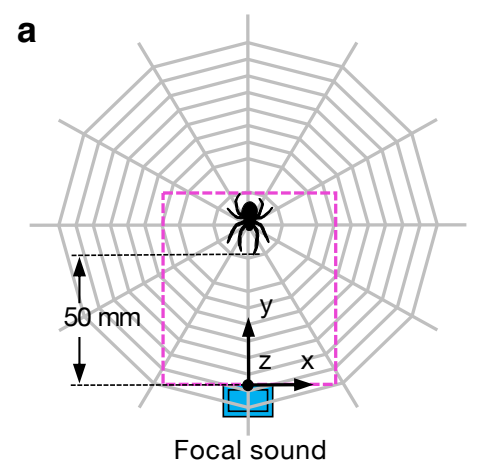

b

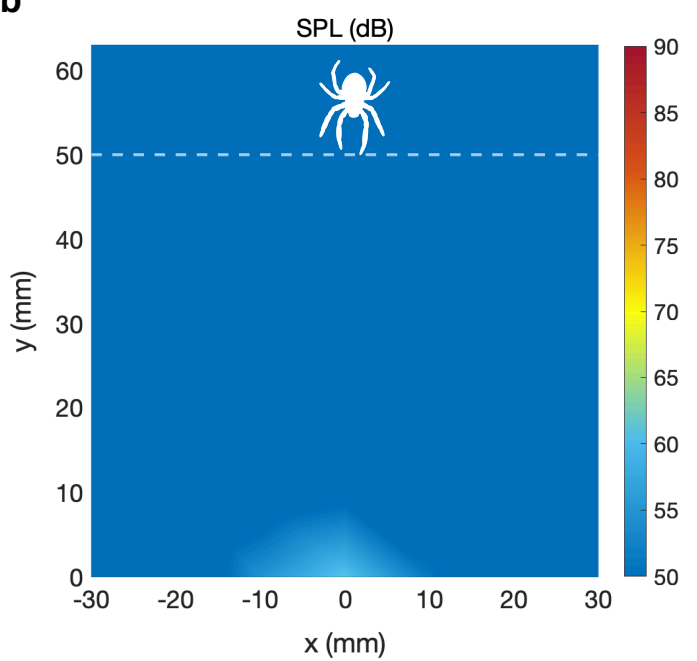

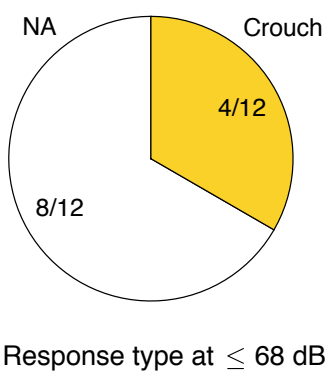

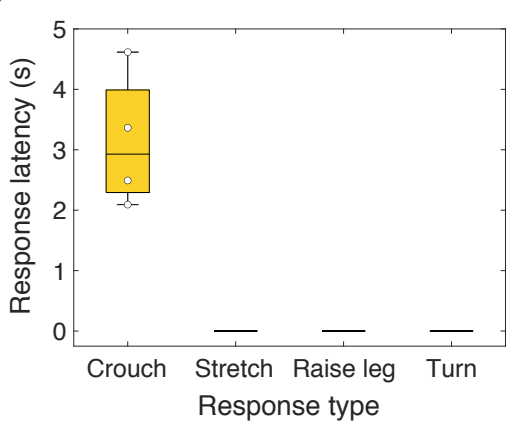

C

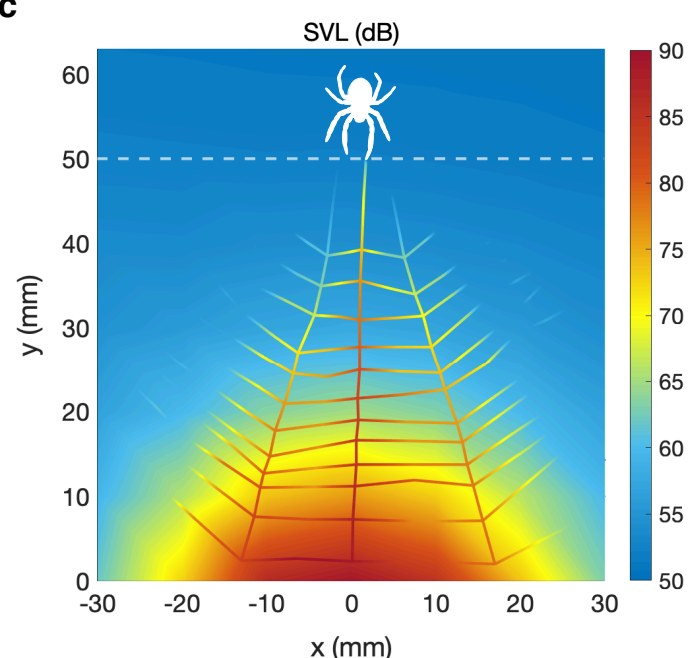

Fig. 3 | Spider behavioral responses to orb-web movements stimulated by focal airborne sound. a, Schematic diagram of setup. Highly localized near-field sound was generated by a miniature speaker, placed as close to the orb-web without touching it, $50 \mathrm{~mm}$ distance in radial distance from a spider resting in its hub web. b, c, Focal airborne sound field at $200 \mathrm{~Hz}$, measured in the orb-web plane as marked by the magenta rectangular region in $\mathbf{a}$. The near-field sound is velocity dominant, where the sound pressure level (b) is almost ignorable in compared with the sound velocity level (c). Minispeaker airborne acoustic signals attenuate quickly and fell well below the detection threshold of spiders (see Methods) after propagating to where the spider sat $(<50 \mathrm{~dB})$, while the induced out-of-plane web movements $(\mathbf{c})$ attenuate less $(\leq 68 \mathrm{~dB})$. d, e, Behavioral response categories and response latencies (each value, median, interquartile and range) to focal tones ( $200 \mathrm{~Hz}$, equivalent $\mathrm{SPL} \leq 68 \mathrm{~dB}, 3 \mathrm{~s}$ duration). 


\section{Methods}

Spiders and their orb-webs. Orb-weaving spiders, Larinioides sclopetarius, were collected from natural habitats in Vestal, N.Y., and kept in laboratory conditions (approx. $22^{\circ} \mathrm{C}$ temperature, a $12 \mathrm{~h}: 12 \mathrm{~h}$ lightdark cycle), where they spontaneously spin orb-webs within wooden frames $(30 \mathrm{~cm} \times 30 \mathrm{~cm} \times 1 \mathrm{~cm})$ in transparent enclosures with fruit flies (Drosophila). After weaving the orb-webs, spiders positioned themselves to settle within the hub region of the orb-webs, as in nature. A red dim light was always turned on to enable basic visualization for setting up experiments during all light-dark cycles. Female spiders were used in all experiments. The ranges of body length and weight of spiders are 5-8 $\mathrm{mm}$ and 0.7-1.9 $\mathrm{mN}$, respectively.

Experimental setups. Three kinds of speaker configurations were used to create different airborne sound waves around the orb-web: 1) remote normal-incident sound wave propagating in the direction perpendicular to the plane of the web was generated by a subwoofer (Coustic HT612) and a tweeter (ESS Heil AMT1) with the crossover set at $2 \mathrm{kHz}$, placed $3.0 \mathrm{~m}$ away to the plane of the orb-web as shown in Extended Data Fig. 1a; 2) oblique-incident sound was created by two identical loudspeakers (NSM Model 5), placed $0.50 \mathrm{~m}$ away, $45^{\circ}$ in azimuth on the left and right side of the spider, as shown in Extended Data Fig. 1b; 3) focal sound was generated by a miniature speaker (CUI CMS-15118D-L100, dimensions 15 $\mathrm{mm} \times 11 \mathrm{~mm} \times 3 \mathrm{~mm}$ ), placed close to the web without touching (about $2 \mathrm{~mm}$ distance to the orb-web plane), $50 \mathrm{~mm}$ in radial distance from the spider resting in its hub web as shown in Fig. $3 \mathrm{a}$.

The sound pressure $p(t)$ around the orb-web was measured by a calibrated microphone (B\&K 4138) placed close to the orb-web without touching. The out-of-plane motion of the orb-web was measured by a laser Doppler vibrometer (Polytec OFV-534). To measure different locations, the laser Doppler vibrometer was mounted on a precise 2-dimensional linear stage (Newport ILS250PP), controlled by a motion controller (Newport model ESP 301). The built-in camera within the vibrometer enabled the visualization of the measurement position on the orb-web. Base vibration was measured with a tri-axial accelerometer (PCB 356A11). A data acquisition system (NI PXI-1033) was used to acquire data. Spider behavioral responses were recorded using a video camera of $60 \mathrm{fps}$.

Testing of spider behavioral response to airborne sound. The spiders' behavioral reactions to airborne, tonal stimuli were videotaped under 3 acoustic conditions: 1 ) normal-incident sound waves ( $\mathrm{N}=48$ spiders), 2) oblique-incident directional sound waves ( $\mathrm{N}=12$ spiders), and 3 ) small focused beams of sounds ( $\mathrm{N}=12$ spiders). Detailed experimental configurations are listed in Supplementary Table 1. Before making any measurements, we ensured that spiders were undisturbed and resting in the hub of their orb-webs. To guarantee this, after gently placing it in the testing area, the spider was left alone in the anechoic chamber for $0.5 \mathrm{~h}$ before playing tonal stimulation. If a spider responds with multiple behaviors to stimuli, we counted only the initial response when parsing among categories of behavior. At $88 \mathrm{~dB}$ stimulation, we noted that the spider's body posture sometimes resulted in a very slight rotation, so we labeled such behavior as a turn only when a spider's turn angle was unambiguously greater than 10 degrees. The response latency of an individual spider was counted from the recorded video frame number starting from the beginning of the stimuli. For behavior reactions to the oblique-incident sound, while a complete audio trial contains 4 sub clips (Extended Data Fig. 1d), we counted only the probability of successful source localization of spiders in response to the first sub audio.

Characterization of the normal-incident sound field. By placing speakers far away $(3.0 \mathrm{~m})$ from the spider web in our anechoic chamber (Extended Data Fig. 1a), we approximately created a normal-incident planar acoustic field. The direction of the propagation of the sound waves was roughly perpendicular to 
the plane of the orb-web. Extended Data Fig.1e shows an example of the measured sound pressure level (SPL) at $200 \mathrm{~Hz}$ around the spider orb-web in an area of $240 \mathrm{~mm} \times 240 \mathrm{~mm}$ with scanned gap distance 10 $\mathrm{mm}$, which is considerably uniform with a SPL variation within $1 \mathrm{~dB}$ at different locations. The broadband SPL at all measured locations is shown in Extended Data Fig. 1f, which has a variation within $2 \mathrm{~dB}$ under the measured frequency. The sound field around the spider web can be regarded as a plane wave approximately, considering the little variation of SPL. For a plane sound wave, the air particle velocity $u(t)$ nearby the orb-web can be determined by measuring the sound pressure $p(t)$ according to $u(t)=p(t) / \rho_{0} c$, where $\rho_{0}$ is the density of air, $\mathrm{c}$ is the speed of sound in air. The sound pressure level (SPL) was calculated by $S P L=20 \log _{10}\left(P_{R M S} / P_{r e f}\right)$, where $P_{R M S}$ is the root mean square of the measured sound pressure $p(t)$, $\mathrm{P}_{\text {ref }}=20 \mu \mathrm{Pa}$ is the reference pressure.

Measurement of the airborne acoustic responsivity of the orb-webs. We characterized the airborne acoustic responsivity of the orb-webs under well-controlled normal-incident plane-wave sound field as shown in Extended Data Fig. 1a. To precisely characterize the frequency responses, we used a short period of pure tones at various frequencies from $100 \mathrm{~Hz}$ to $10000 \mathrm{~Hz}$. The measured data was then processed at each frequency with Least Squares Data Fitting to estimate the amplitude and phase of the measured airborne acoustic waves from microphone and the motion of the web threads measured by the laser vibrometer. To measure the velocity responses of a spider and its peripheral orb-web, it's crucial to keep the spider stable during the measurement process. To avoid the motion interruption from the behavioral responses, we stimulated a spider with acoustic tones $(200 \mathrm{~Hz}, 88 \mathrm{~dB}, 3 \mathrm{~s}$ duration) before frequency measurement. After several cycles of acoustic stimulus, a spider rarely responded to the stimuli and kept stable so the measurement could be completed.

Mapping the orb-web motion induced by airborne sound. We mapped the out-of-plane motion of the orb-web at different locations with and without the spider resting in hub web respectively, and then recreated the motion based on the measured velocity response of web threads at different locations under a certain measurement frequency f. Suppose the air particle velocity is $\mathrm{u}(\mathrm{t})=\mathrm{U} \mathrm{e}^{\mathrm{i} \omega \mathrm{t}}$, the motion of a web position can be expressed as $v(t)=V e^{i \omega t}$, where $\omega=2 \pi f$, $U$ and $V$ are complex amplitudes. Since the air particle velocity around all measured web threads can be approximately regarded to be uniform in the normal-incident plane-wave sound field, the complex amplitude of the air particle velocity nearby all measured web positions can be expressed as $\mathrm{U}=|\mathrm{U}| \mathrm{e}^{\mathrm{i} \varphi}$, where $\varphi$ is the phase. Supposed the measured complex amplitude at a web position $n$ is $V_{n}=\left|V_{n}\right| e^{i \varphi_{n}}$, the time response of the measured point to a steady-state airborne sound can be expressed as $v_{n}(t)=\left|V_{n}\right| e^{i \varphi_{n}} e^{i \omega t}$. As the measured frequency responses of web threads at different locations contain the velocity amplitude as well as phase, these results can be used to create the steady-state motion of the measured objects. Figures and videos (Fig. 1a, Fig. 1d, and Supplementary Video S3) demonstrating the out-of-plane motion of the spider and its orb-web were created by STAR 7, a commercial modal analysis software.

Characterization of the focal sound field. We characterized the airborne signals as well as the out-ofplane transverse motion of web strands induced by the miniature speaker. Both sound pressure level (SPL, Fig. 3b) and sound velocity level (SVL, Fig. 3c) of the sound field were characterized. We scanned the acoustic pressure field by a probe microphone. The acoustic velocity field was characterized by an easily made velocity probe, constituted by the laser Doppler vibrometer and a strand of spider silk ${ }^{7}$. The spider silk has a sub-micron diameter, $5 \mathrm{~mm}$ length, and was supported at its two ends loosely. By focusing the laser beam perpendicular to the longitudinal direction of the spider silk at its middle position, we measured the silk motion induced by the motion of the air particles. Before scanning of the velocity field in the orbweb plane, we confirmed that the silk velocity probe representing the air particle motion closely (i.e. 
$\left.361 \mathrm{~V}_{\text {silk }} / \mathrm{V}_{\text {air }} \sim 1\right)$ in the measured frequency, as shown by the insert figure in Supplementary Data Fig. 5a. Since 362 velocity is a vector, we scanned the acoustic particle velocity in 3 dimensions, including $V_{x}, V_{y}$ and $V_{z}$. 363 The overall amplitude of velocity $V$ (Fig. 3c) at a position was evaluated by $V=\left(V_{x}^{2}+V_{y}^{2}+V_{z}^{2}\right)^{-1 / 2}$. To 364 compare between the air particle velocity and sound pressure, the sound velocity level (SVL) was 365 calculated by $S V L=20 \log _{10}\left(V_{\mathrm{RMS}} / \mathrm{V}_{\text {ref }}\right)$, where $\mathrm{V}_{\mathrm{RMS}}$ is the root mean square of the measured particle 366 velocity $\mathrm{V}, \mathrm{V}_{\text {ref }}=\mathrm{P}_{\text {ref }} / \rho_{0} \mathrm{c}$ is the reference velocity.

367 Before propagating to the location of spider, the nearfield airborne signals fell well below the detection 368 threshold of the spiders ${ }^{10,20}$. The SPL (Fig. 3b) was below $30 \mathrm{~dB}$, while the SVL (Fig. 3c) was lower than $36950 \mathrm{~dB}\left(\mathrm{~V}_{\mathrm{rms}}<0.016 \mathrm{~mm} / \mathrm{s}\right)$ after reaching to the spider. The ultralow airborne signal is even lower than the 370 detection threshold of the jumping $\operatorname{spider}^{20}$, which enables the best-known spider sensitivity $(\sim 65 \mathrm{~dB})$ so far. We never observed any behavioral response of spiders to $50 \mathrm{~dB}$ normal-incident airborne stimuli.

The out-of-plane transverse motion of web strands induced by the mini-speaker attenuated slower than the near-field airborne signals, so as to transmit the local vibrational signals to the spider (Fig. 3c and Supplementary Data Fig. 5d). Since the initial SVL generated by the mini-speaker $50 \mathrm{~mm}$ away from the spider is about $88 \mathrm{~dB}$, and it attenuates about $20 \mathrm{~dB}$ after $40 \mathrm{~mm}$, the equivalent SPL of the vibrational signals transmitted to the spider is less than $68 \mathrm{~dB}$. 
377 Acknowledgments We thank Charles Walcott for his expert consultation and advice. This work was 378 supported in part by the National Institute on Deafness and Other Communication Disorders of the 379 National Institutes of Health under Award Number R01DC017720 (to R.N.M).

380 Author contributions Conceptualization: J.Z, R.R.H, R.N.M; Methodology: J.Z, J.L, G.M, J.A.S, C.I.M, 381 R.R.H, R.N.M; Investigation: J.Z, J.L, G.M, J.A.S; Formal Analysis: J.Z, J.L, G.M, J.A.S, C.I.M, R.R.H, 382 R.N.M; Resources: C.I.M, R.R.H, R.N.M; Funding acquisition: R.N.M; Writing - original draft: J.Z, 383 R.R.H; Writing - review \& editing: J.Z, J.L, G.M, J.A.S, C.I.M, R.R.H, R.N.M; Supervision: R.R.H, 384 R.N.M.

385 Competing interests The authors report no conflict of interest.

386 Data and materials availability All data are included in this published article (and its supplementary 387 information files).

388 Additional information

389 Supplementary Information is available for this paper.

390 Supplementary Materials:

391 Supplementary Text

$392 \quad$ Supplementary Table 1

$393 \quad$ Supplementary Table 2

$394 \quad$ References

395 Other Supplementary Materials:

$396 \quad$ Supplementary Movie 1

$397 \quad$ Supplementary Movie 2

$398 \quad$ Supplementary Movie 3

$399 \quad$ Supplementary Audio 1 


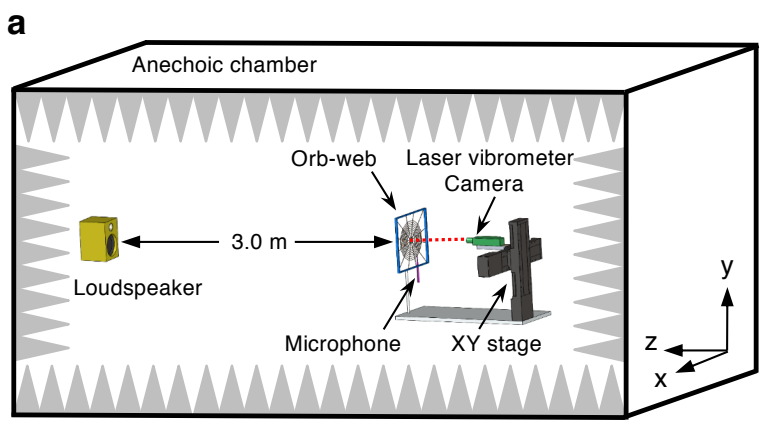

Normal-incident sound

c
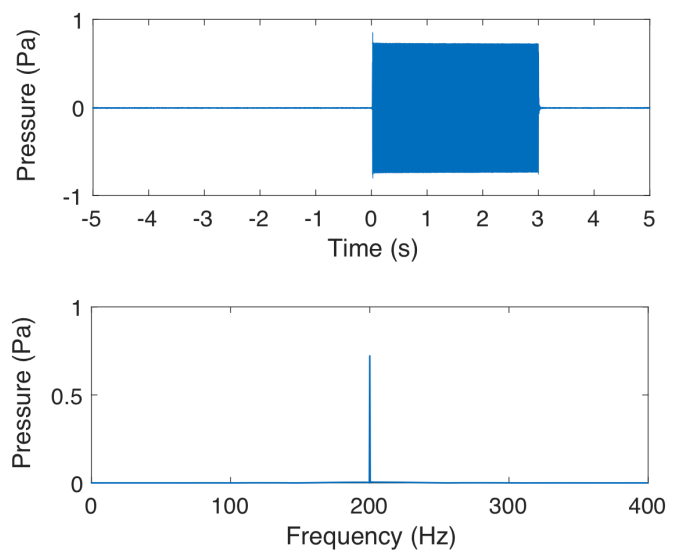

e

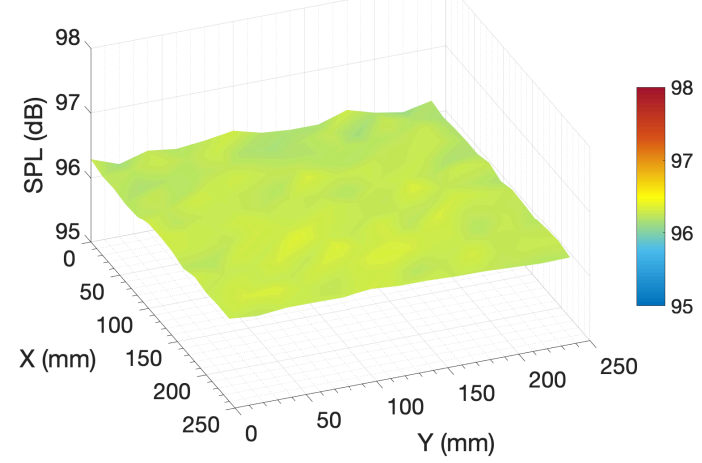

b

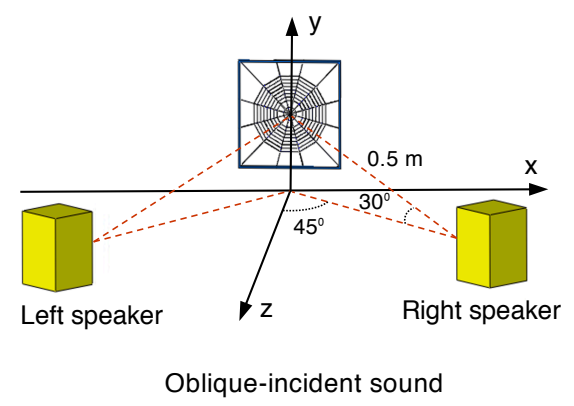

d
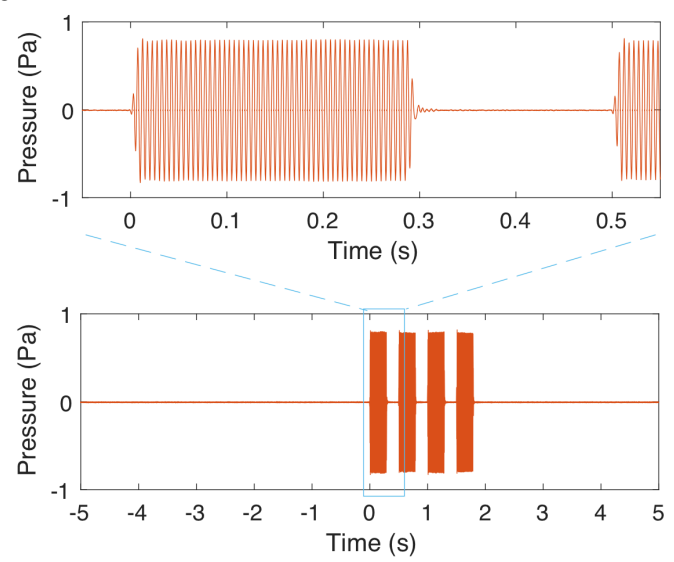

f

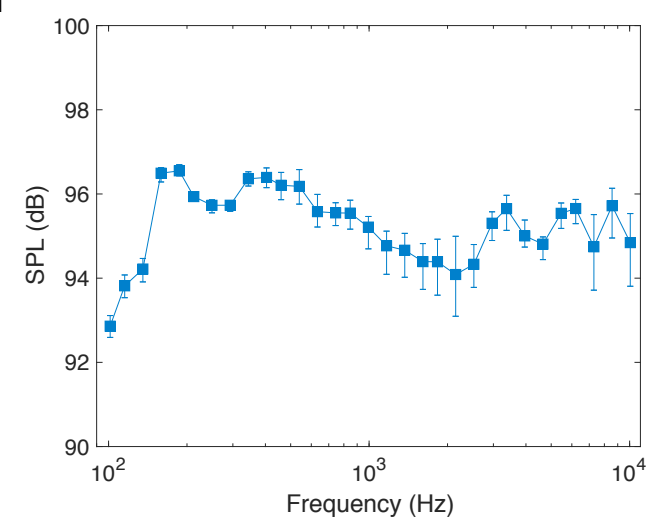

Extended Data Fig. 1 | Generation of remote airborne sound. a, b, Schematical diagrams of the normalincident and oblique-incident sound setups. c, An example of the normal-incident acoustic stimuli (200 $\mathrm{Hz}, 88 \mathrm{~dB}, 3 \mathrm{~s}$ duration). The top and bottom images show the time traces and the fast Fourier transform (FFT) of the acoustic signals. d, An example of the oblique-incident stimuli. A complete stimuli contains 4 sub directional clips of duration $0.3 \mathrm{~s}(200 \mathrm{~Hz}, 88 \mathrm{~dB})$ with the silent gap of $0.2 \mathrm{~s}$. Individual spider was randomly subjected to one of the patterns of directional acoustic tones, either $\mathrm{L}+\mathrm{R}+\mathrm{L}+\mathrm{R}$ or $\mathrm{R}+\mathrm{L}+\mathrm{R}+\mathrm{L}$, where $\mathrm{L}$ represents the sound generated by the left loudspeaker, while $\mathrm{R}$ represents the sound generated by the right speaker. e, An example of the normal-incident sound fields around the spider orb-web at 200 Hz. f, Normal-incident sound pressure level (SPL) around the orb-web at a wide range of frequencies. The minimum, mean, and maximum SPL are presented. Normal-incident sound field can be regarded as a planewave approximately. 
a

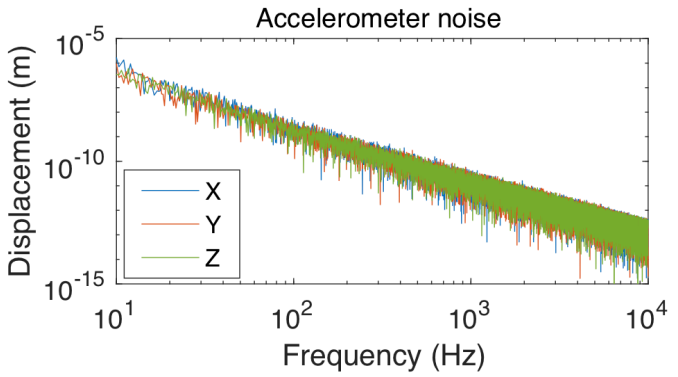

C

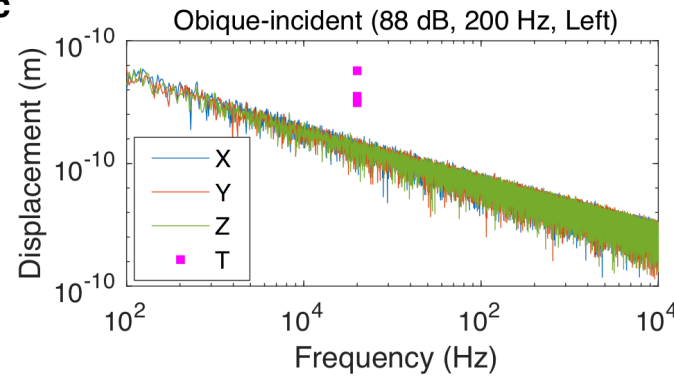

e

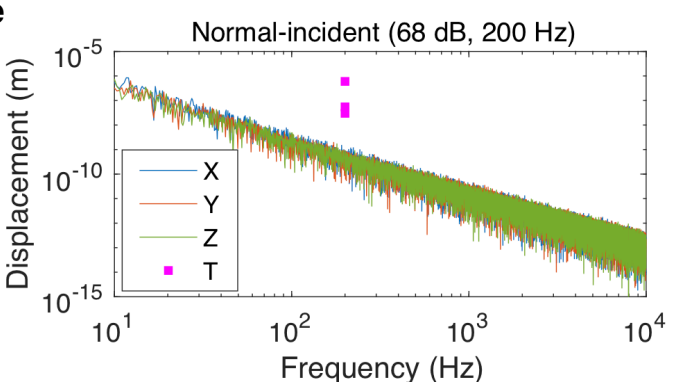

g

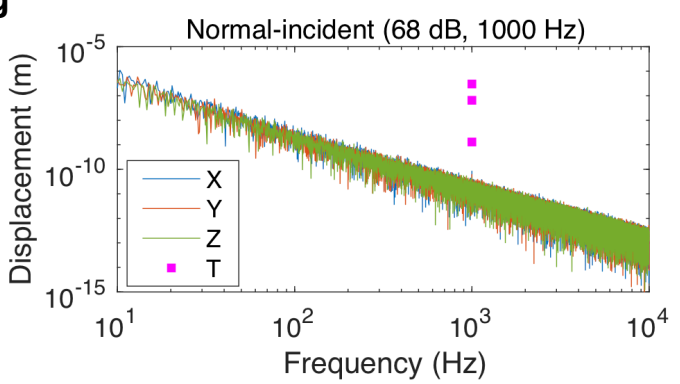

b

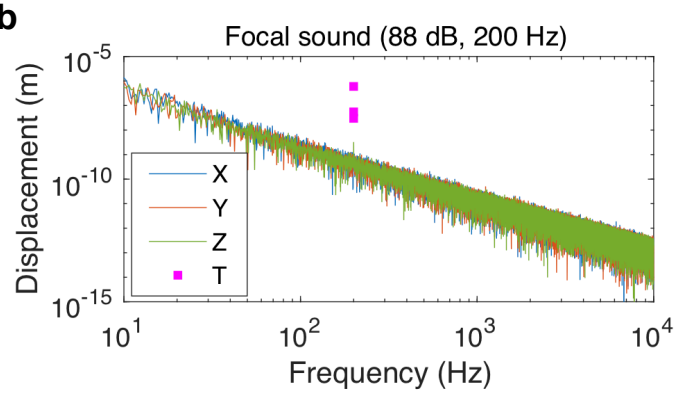

d

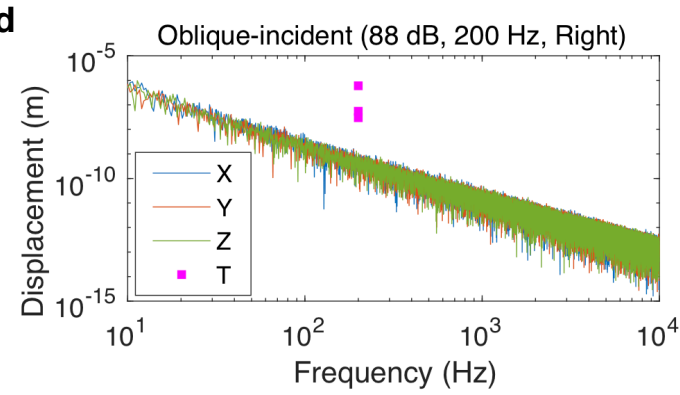

$\mathbf{f}$

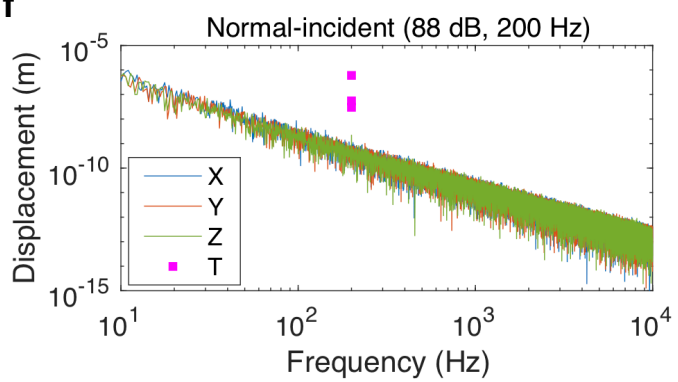

h

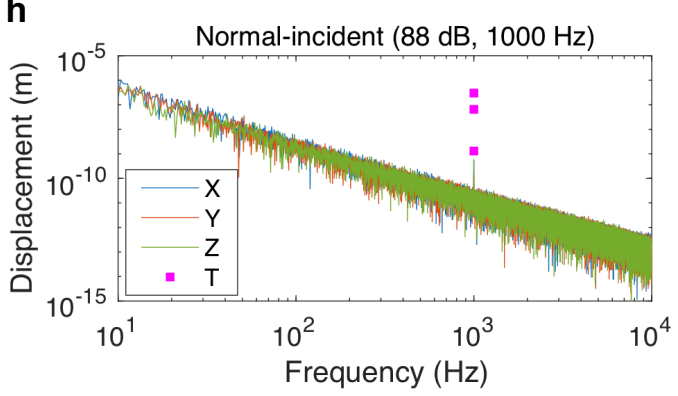

Extended Data Fig. 2 | Speaker-induced base excitations. Excitations are measured at the base of the orb-web supporting frame with a tri-axial accelerometer. a, Noise floor of the accelerometer. b, Base excitations of the focal sound setup. $\mathbf{c}, \mathbf{d}$, Base excitation of the oblique-incident sound setup. e-h, Base excitations of the normal-incident sound setup. In the figure legends, X, Y, Z represent the measured 3dimensional directions, $\mathrm{T}$ represents the reported threshold of spider metatarsal lyriform organ ${ }^{10}$. Except the excitation condition as shown in $\mathbf{h}$, which is more than 2 times $(6 \mathrm{~dB})$ lower, all speaker-induced base excitations are more than 10 times $(20 \mathrm{~dB})$ lower than the threshold of the spider metatarsal lyriform organ. 

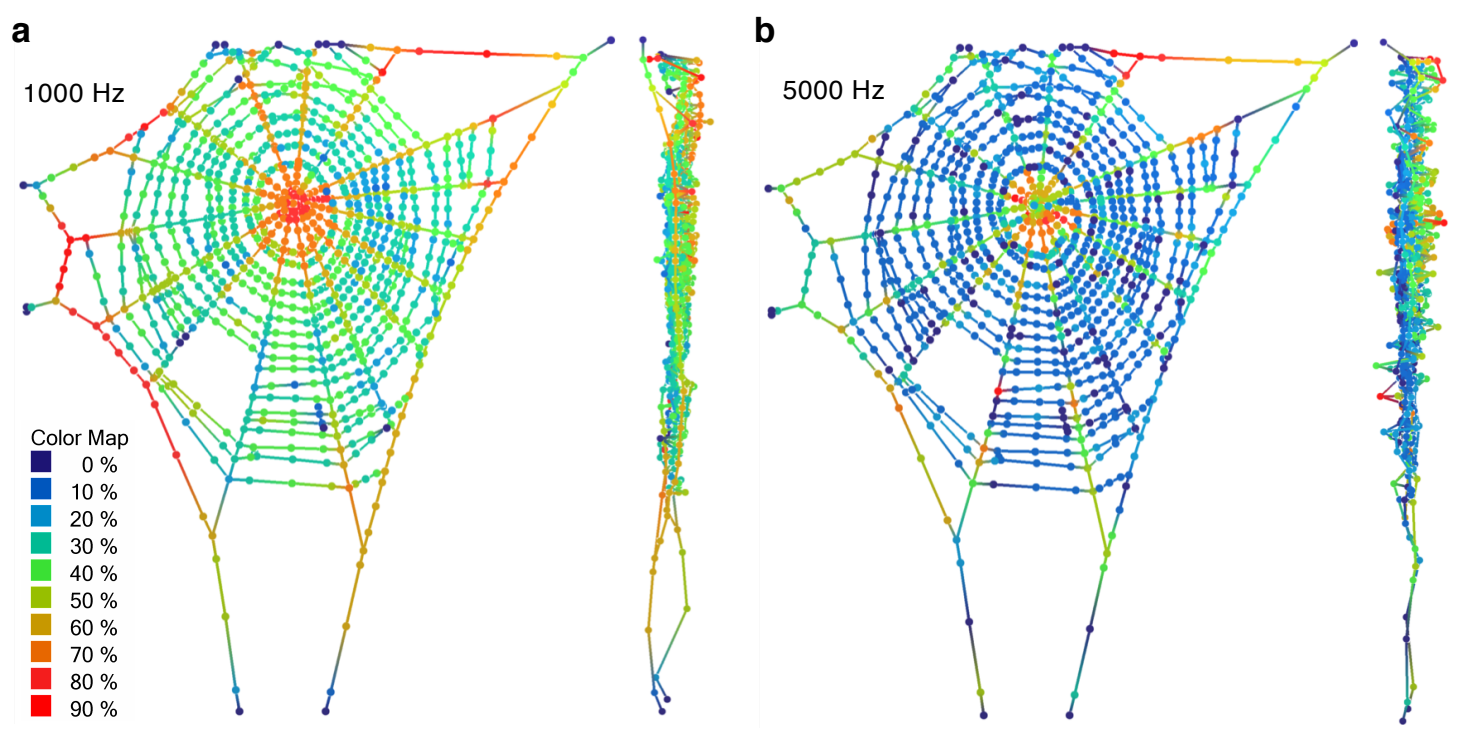

c

d
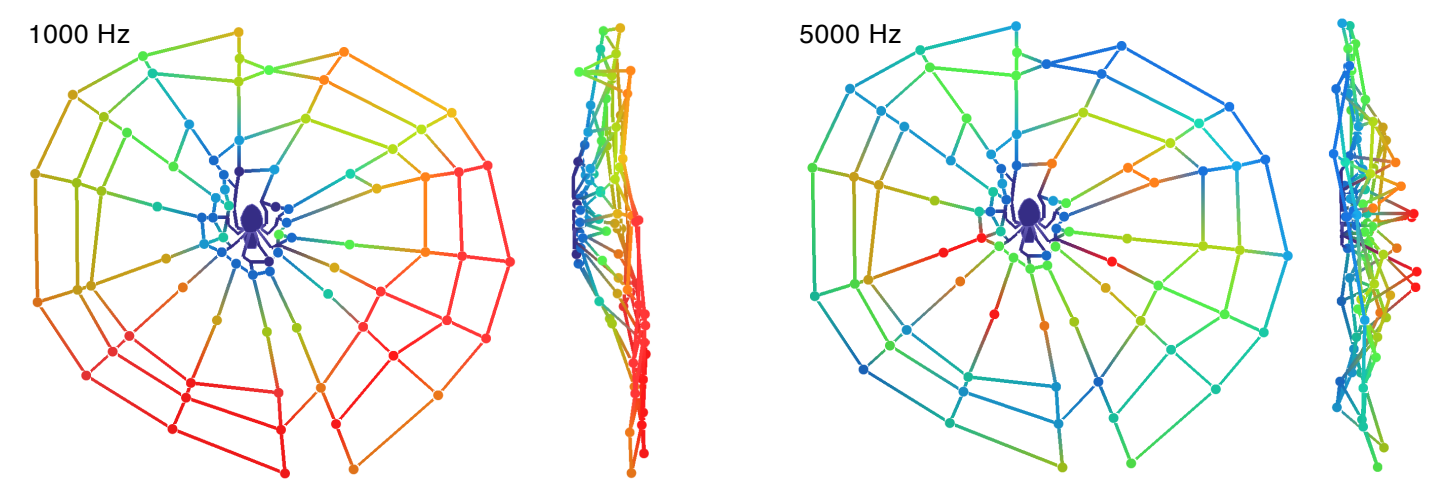

Extended Data Fig. 3 | Orb-web responses to remote normal-incident sound. a, b, Out-of-plane motion of a complete orb-web induced by a steady-state sound field at $1000 \mathrm{~Hz}$ and $5000 \mathrm{~Hz}$. c, d, Out424 of-plane motion of an orb-web containing a live spider, induced by a sound field at $1000 \mathrm{~Hz}$ and $5000 \mathrm{~Hz}$. 425 Color coding of $\mathbf{b}, \mathbf{c}, \mathbf{d}$ same as $\mathbf{a}$. The colored heat map represents the amplitude ratio of the web thread 426 velocity $\mathrm{V}$ to the air particle velocity $\mathrm{V}_{\text {air. }}$. 

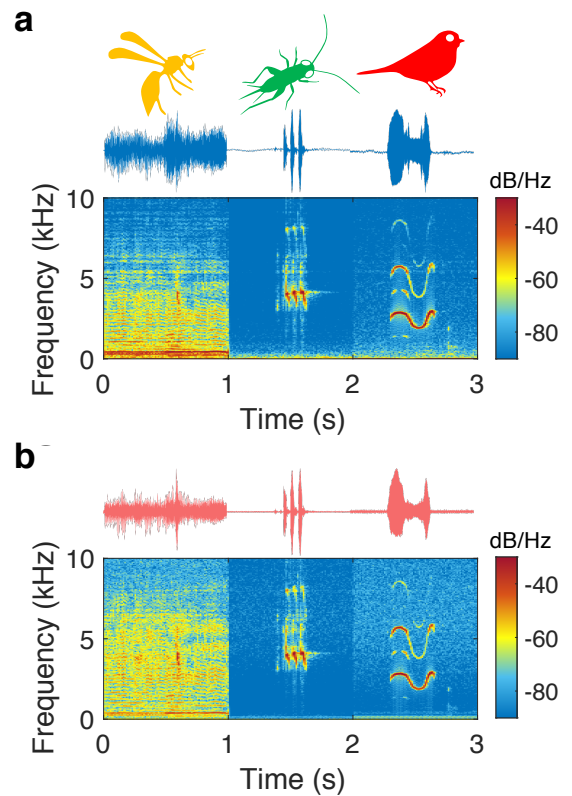

428 Extended Data Fig. 4 | Time-domain traces and spectrograms of the sound-induced orb-web motion 429 containing a live spider. The normal-incident airborne acoustic signal (a) was measured by a pressure 430 microphone nearby the spider. The out-of-plane web motion (b) was measured by a laser vibrometer at a 431 radial web thread, $2 \mathrm{~mm}$ away from the spider foreleg. 
a

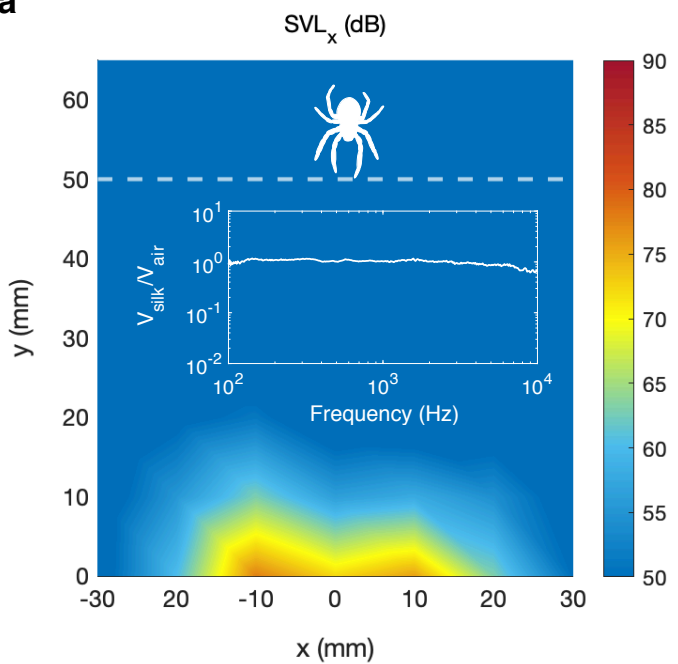

C

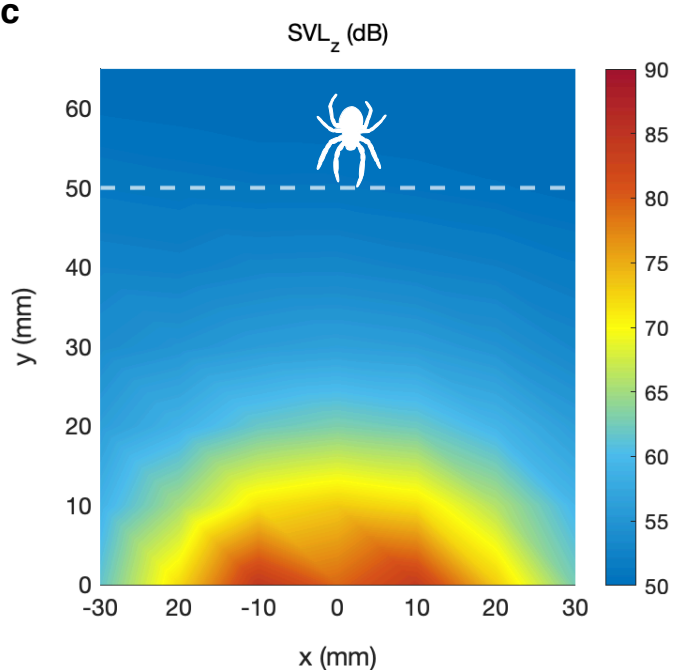

b

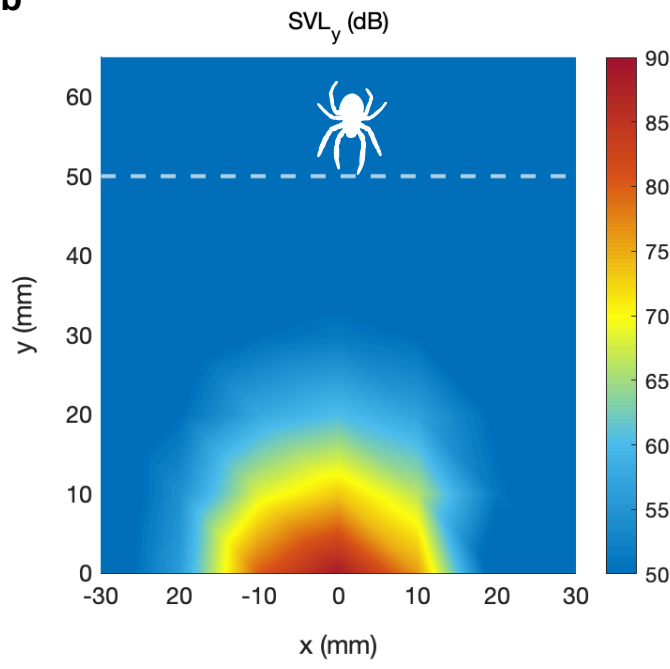

d

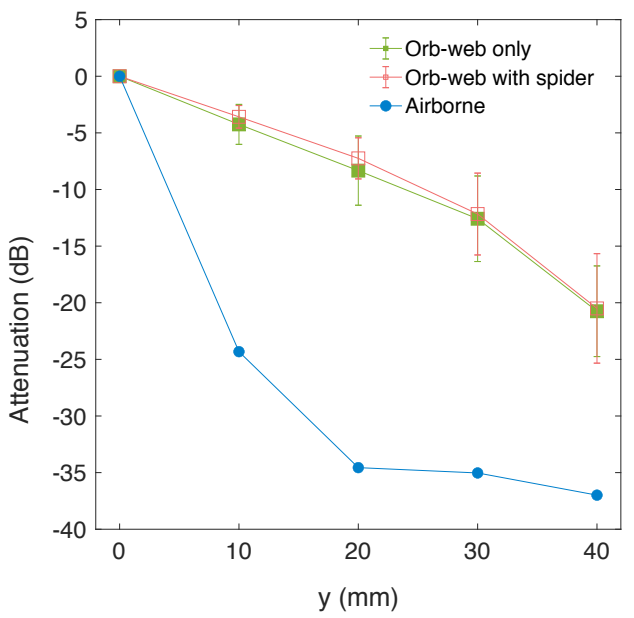

433 Extended Data Fig. 5 | Focal airborne acoustic field generated by the miniature speaker. a-c, 434 Measured sound velocity level (SVL) in 3-dimensions along the plane of the orb-web. The air particle velocity in each dimension was mapped by a silk velocity probe, whose characteristics are shown as the insert figure in a. The 3-dimensional velocity components constitute the overall SVL as shown in Fig. 3c. d, Statistic attenuation of the out-of-plane web vibration along the radial threads of orb-webs $(\mathrm{N}=12$ spiders). Error bars show one standard deviation. The near-field airborne signal is also shown as comparison, extracted from the overall SVL measured at multiple locations (Fig. 3c, $\mathrm{x}=0 \mathrm{~mm}, \mathrm{y}=0 \sim 40$ $\mathrm{mm})$. The out-of-plane motion of web threads induced by the mini-speaker attenuated slower than the airborne signals, so as to transmit the vibrational signals to the spider. 


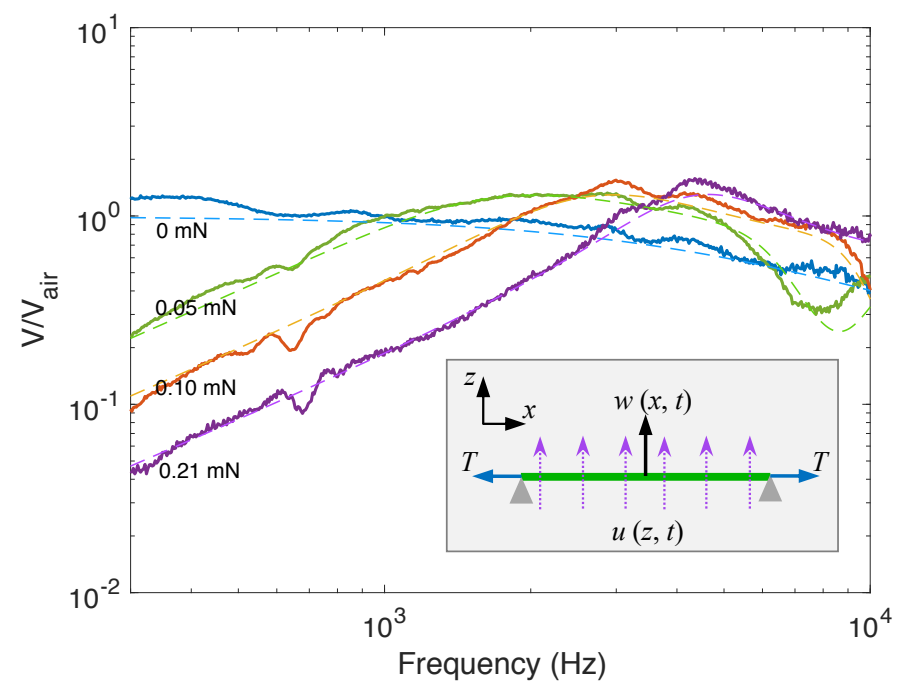

450 of spiders $(0.7-1.9 \mathrm{mN})$. 